\title{
McGinn-White sign resulting from ischemic heart disease: A case report
}

\author{
Rodrigo Moraes Reis*, Josué Almeida Victorino \\ Federal University of Health Sciences of Porto Alegre (UFCSPA), Porto Alegre, Brazil
}

\begin{abstract}
The S1Q3T3 sign, also known as the McGinn-White is classically associated with pulmonary embolism, but not is not a pathognomonic sign. In our case, a 47 years old man with no history of known comorbities was taken to the emergency department by ambulance in a cardiac arrest. The return of spontaneous circulation occurred in aroud 50 minutes (after 35 minutes of cardiopulmonary resuscitation. The electrocardiogram showed sinus rhythm with a s1q3t3 pattern. Laboratory tests revealed a troponin I rise from 1,25 ug/L to 31,02 ug/L. Subsequently the patient underwent cardiac catheterization that showed: anterior descending artery with proximal obstruction of $90 \%$, first and second diagonal branch with proximal obstruction of $80 \%$, left circumflex artery with proximal obstruction of $90 \%$ and a dominant right coronary artery occluded in the middle third of the artery. Therefore, it was decided to undergo myocardial revascularization surgery. He presented good clinical evolution and was discharged from the hospital with outpatient return.
\end{abstract}

\section{Background}

Sudden cardiac arrest (SCA) is the sudden hemodynamic collapse, typically due to malignant arrhythmias. These events are more common in patients with structural heart disease, particularly coronary heart disease.

The electrocardiogram (ECG) should be readily performed during the etiological investigation because It is a noninvasive, low cost and also widely available in areas with scarcity of resources. It may give some clues to the etiology of the SCA.

The S1Q3T3 sign, also known as the McGinn-White sign was described in 1935 by McGinn and White. It has the following voltage criteria: S wave in lead I and Q wave in lead III, and amplitude of more than $0.15 \mathrm{mV}(1.5 \mathrm{~mm})$ associated with inversion of the $\mathrm{T}$ wave in lead III. It is classically related to pulmonary embolism (PE), but not pathognomonic of it.

\section{Case report}

Male of 47 years old with no history of known comorbidities was taken to the emergency department by ambulance in a cardiac arrest. According to the paramedics, the response time was of 15 minutes, in which the patient's wife was oriented to perform cardiopulmonary resuscitation $(\mathrm{CPR})$.

The rhythm was of ventricular fibrillation. CPR was performed for around 35 minutes by paramedics until the return of spontaneous circulation, there was performed orotracheal intubation and defibrillation maneuvers. It was administered amiodarone and adrenaline. Patient arrived intubated with a orotracheal tube and stable vital signs. He presented a transient depressed level of consciousness, then underwent mechanical ventilation at the ICU. There was no history of illicit drug use but he did have a history of smoking since he was 18 years old.

An electrocardiogram was performed on arrival. It revealed a right bundle branch block and atrial fibrillation, with a ventricular frequency of 120 beat per minute. A transthoracic echocardiography was performed. It shows a moderate left ventricular segmental contractile dysfunction with an ejection fraction of $38 \%$, diffuse hypokinesia, and posterior wall akinesia. Laboratory tests revealed a troponin I rise from $1,25 \mathrm{ug} / \mathrm{L}$ to $31,02 \mathrm{ug} / \mathrm{L}$. Then the electrocardiogram was repeated and showed sinus rhythm with a s1q3t3 pattern (Figure 1).

Subsequently the patient underwent cardiac catheterization that showed: anterior descending artery with proximal obstruction of $90 \%$, first and second diagonal branch with proximal obstruction of $80 \%$, left circumflex artery with proximal obstruction of $90 \%$ and a dominant right coronary artery occluded in the middle third of the artery.

Therefore, it was decided to undergo myocardial revascularization surgery. Saphenous revascularization was performed for first and second diagonal branches, circumflex artery and posterior descending artery. A mammary artery bypass was performed for the anterior descending artery (Figure 2). He presented good clinical evolution and was discharged from the hospital with outpatient return.

\section{Discussion}

The S1Q3T3 sign, also known as the McGinn-White sign was described in 1935 by McGinn and White. It has the following voltage criteria: $\mathrm{S}$ wave in lead I and Q wave in lead III, and amplitude of more than $0.15 \mathrm{mV}(1.5 \mathrm{~mm})$ associated with inversion of the $\mathrm{T}$ wave in lead III.

This signal is present in less than $10 \%$ of cases of PE, generally indicating a worse prognosis because it represents right ventricular dysfunction [1]. In a observational cohort study of a emergency

${ }^{*}$ Correspondence to: Rodrigo Moraes Reis, Federal University of Health Sciences of Porto Alegre (UFCSPA), Sarmento Leite Street, 245 - Porto Alegre - RS, CEP 90050-170, Brazil, E-mail: rodrigomoraesreis@gmail.com

Received: June 03, 2019; Accepted: June 17, 2019; Published: June 20, 2019 


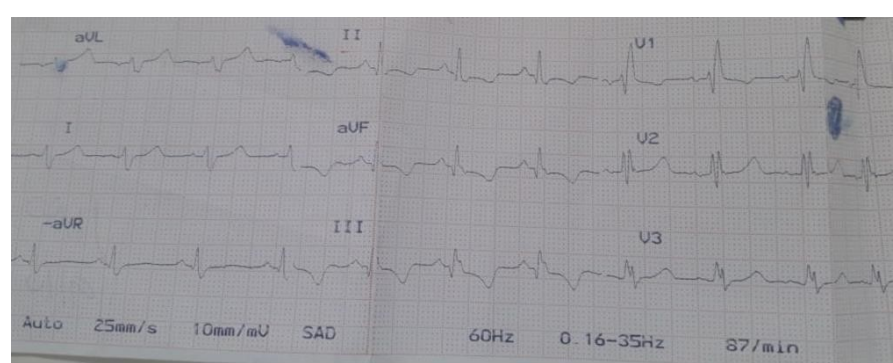

Figure 1. S1Q3T3 pattern on ECG.

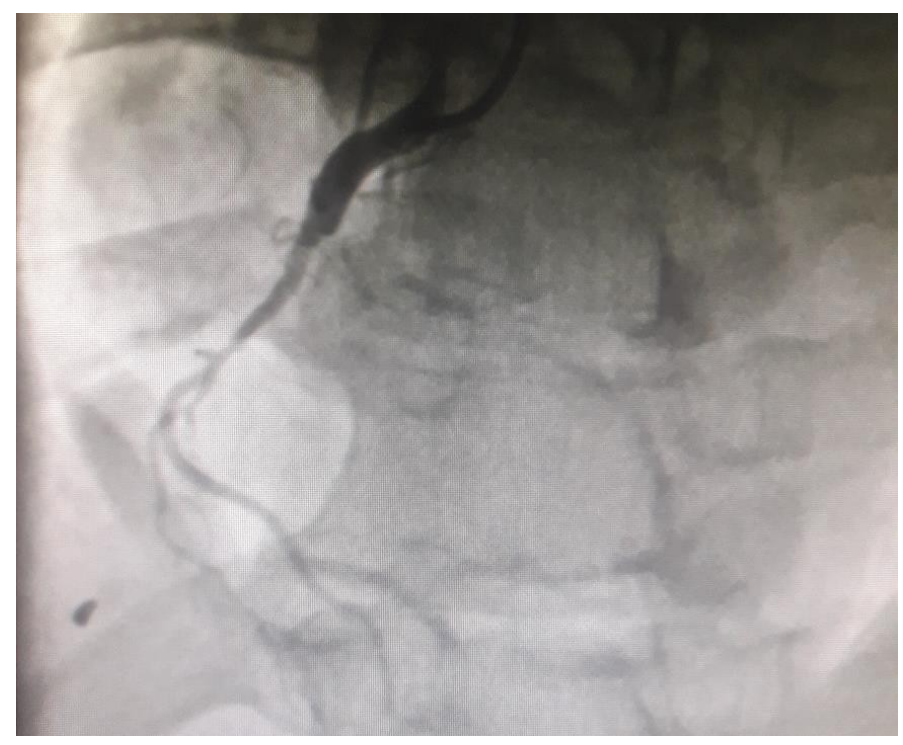

Figure 2. Cardiac catheterization with ocluded right coronary artery in the middle third.

department, in the patients who were tested for pulmonary embolism, the S1Q3T3 pattern was found in $8.5 \%$ of the patients with confirmed $\mathrm{PE}$ and 3.3\% without PE [2]. The S1Q3T3 pattern is usually related to $\mathrm{PE}$, however, this signal is neither sensitive nor specific for PE and may be present in diseases that occur with right ventricular dysfunction.
Although right ventricular myocardial infarction (RVMI) as a myocardial infarction involving only right ventricle is an uncommon event, the impairment of the right ventricle is an independent predictor of major complications and in- hospital mortality. Poor outcome is usually related to profound hemodynamic and electrical complications, which occur in approximately $50 \%$ of affected individuals [3]. A dramatic presentation of RVMI is the SCA.

The incidence of SCA increases with age and with underlying cardiac disease. In addition, men are two to three times more likely to experience SCA than women. [4]. Seventy percent of SCA cases are thought to be due to ischemic coronary disease. Other causes include congestive heart failure, left ventricular hypertrophy, intracranial hemorrhage and pulmonary embolism.

Promptly performed CPR is the main determinant of prognosis. In our case, the patient's spouse was instructed to perform CPR, minimizing the damages of the absence of spontaneous circulation, mainly cerebral. Our patient had posterior wall akinesia on the echocardiogram responsible by the ECG findings due to the occlusion of the right coronary artery showed in the cardiac catheterization.

\section{Conclusion}

The S1Q3T3 pattern is generally related to PE, however, many other conditions that affect the right ventricle may also present this ECG finding. Our patient had posterior wall akinesia due to a myocardial infarction from a right coronary artery occlusion.

\section{References}

1. Marchick MR, Courtney DM, Kabrhel C, Nordenholz KE, Plewa MC, et al. (2010) 12-lead ECG findings of pulmonary hypertension occur more frequently in emergency department patients with pulmonary embolism than in patients without pulmonary embolism. Ann Emerg Med 55: 331-335. [Crossref]

2. Qaddoura A, Digby GC, Kabali C, Kukla P, Zhan ZQ, et al. (1993) Right ventricula infarction as an independent predictor of prognosis after acute inferior myocardial infarction. $N$ Engl J Med 328: 981-988. [Crossref]

3. Siscovick DS (1993) Challenges in cardiac arrest research: data collection to assess outcomes. Ann Emerg Med 22: 92-98. [Crossref]

4. Podrid PJ (2019) Overview of sudden cardiac arrest and sudden cardiac death. Post TW, ed. Waltham, MA: UpToDate Inc. https://www.uptodate.com (Accessed on March 03, 2019.).

Copyright: (C2019 Reis RM. This is an open-access article distributed under the terms of the Creative Commons Attribution License, which permits unrestricted use, distribution, and reproduction in any medium, provided the original author and source are credited. 